\title{
Influence of Meshing on the Numerical Simulation of Liquid Composite Molding Processes
}

\begin{abstract}
Computer simulation has been an efficient and cost-effective tool for the Liquid Composite Molding (LCM) processes, including the RTM, VARTM, and resin infusion, compared to trialand-error. The Control Volume Finite Element Method (CVFEM) has been the predominant method for simulation. Two critical issues of CVFEM are simulation accuracy and computational efficiency, and they are strongly dependent on meshing. In this paper, the influence of meshing on the simulation accuracy is investigated. Both uniform and non-uniform meshes are studied. The results show that for a radial flow, simulation accuracy can be significantly improved by using non-uniform meshes. A case study is conducted and it is shown that for a point injection, the computation time for mold filling simulation can be reduced by more than $99 \%$ while maintaining the same simulation accuracy.
\end{abstract}

Keywords: Composite; mold filling; simulation

\section{Introduction}

Because of the relatively low equipment and tooling costs, short cycle times and excellent design flexibility, liquid composite molding (LCM) processes, which include resin transfer molding (RTM) and vacuum assisted resin transfer molding (VARTM) processes, have attracted considerable attention and gained the status as a preferred method for manufacturing structural composite components. Complete filling of the mold with adequate wetting of the fibrous preform is critical in LCM. Incomplete impregnation in the mold leads to defective parts containing dry spots. In order to achieve good quality, processing parameters such as the locations and numbers of gates and vents need to be well defined when designing the mold.

Traditionally, trial-and-error techniques are widely applied in the composites industry, which is very costly and time consuming. With the development of computing technology, simulation has become a powerful tool for the process design and optimization. A mathematical model for the LCM mold filling consists of a set of partial differential equations and its solution is usually found by numerical methods including finite difference method (FDM), finite element method (FEM) and boundary element method (BEM). FDM was the first attempt used to simulate a two-dimensional RTM process (Coulter,Guceri 1988). By comparing with experimental results, it was proven that due to edge effects, the computing errors were over a reasonable range, which limited further application. Um et al. (Um,Lee 1991) studied two-dimensional flat molds where the permeability and the resin viscosity were constant applying the BEM and concluded that it took less time to generate mesh at each time step than required 
by FDM or FEM. Yoo et al. (Yoo,Lee 1996) and Osswald et al. (Osswald,Tucker 1987) determined that under the limitations of simple geometry parts and isothermal Newtonian problems, the BEM method gave very accurate simulation results.

When FEM is used, mold filling simulation is based on a time dependent solution of an unsteady boundary value problem. Because of the evolving boundary, it is difficult to generate a fixed mesh suitable for all the successive calculation steps of a filling simulation. Although some re-meshing algorithms have been developed (Béchet et al. 2004; Chang,Kikuchi 1994), the Control Volume Finite Element Method (CVFEM) has been the predominant method for process simulation (Bruschke,Advani 1990; Joshi 2000; Liu 2000; Šimáček 2004; Young et al. 1991). It forms and solves a set of equations for nodal control volumes as if they were finite elements. Mesh regeneration is not required, which makes the computation more efficient. Three major steps are needed in the CVFEM flow simulation: (1) use the FE solution to obtain the pressure distribution in the resinfilled region; (2) calculate the resin flow rates; and (3) trace the resin flow front (Joshi 2000).

A critical problem in the optimization using the CVFEM based mold filling simulation is the accuracy of simulation. Because of the nature of FEM, the mesh density has a significant influence on the simulation result. In addition, the definition of injection gate is also an important factor. Traditionally, the injection gates are defined by a single node and the pressure or flow rate boundary condition is applied. The omission of the inlet radius in the model results in a mathematical singularity as the mesh gets refined. Some studies have been conducted to address this issue. Modi et al. (Modi et al. 2003) discussed the influence of gate definition on the flow-front approximation. Šimáček and Advani (Šimáček,Advani 2004) introduced the gate elements for mold filling simulation which employed modified shape functions. Dong (Dong 2007) presented the gate definition methods for 2-D and 3-D mold filling simulations. In this paper, the influence of meshing on the simulation accuracy is investigated. Both uniform and non-uniform meshes are studied. The results show that for a radial flow, simulation accuracy can be significantly improved by using nonuniform meshes. A case study is conducted showing that for a point injection, the computation time for mold filling simulation can be significantly reduced while maintaining the same simulation accuracy.

\section{Uniform Meshing}

\subsection{Channel Flow}

The flow fronts of a channel flow are a series of straight lines. Thus, the flow can be considered as 1-D. From the continuity,

$$
\phi v=-\frac{K}{\mu} \frac{d p}{d x}=C
$$

where $\phi$ is porosity; $v$ is velocity; $K$ is permeability; $\mu$ is dynamic viscosity; $p$ is pressure; and $x$ is 1-D coordinate. After applying the boundary conditions $p=p_{0}$ at the injection gate and $p=0$ at the flow front, Eqn. 1 becomes

$$
\phi v=\frac{K}{\mu} \frac{p_{0}}{x}
$$


Eqn. 2 suggests that $p$ decreases linearly from $p_{0}$ at the gate to 0 at the flow front, as shown in Figure 1.

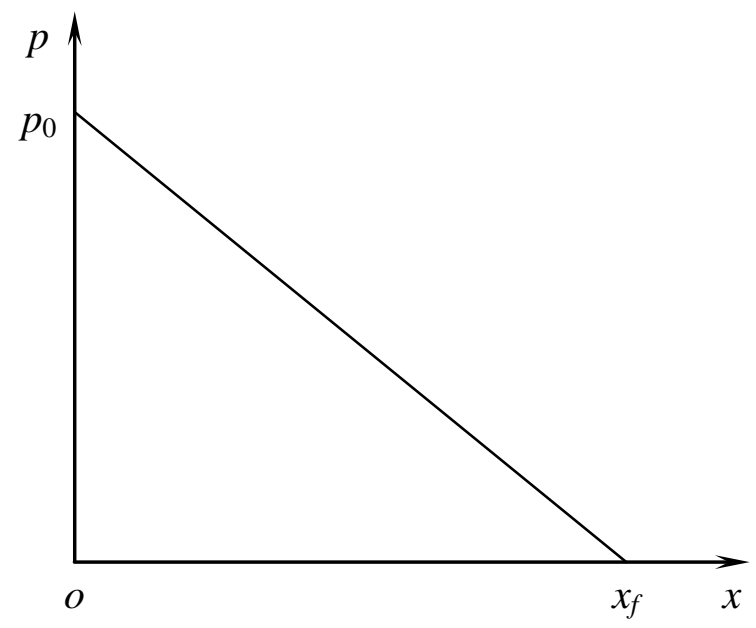

Figure 1: Pressure field of channel flow

Eqn. 2 can be non-dimensionalized as

$$
v^{*}=\frac{1}{x^{*}}
$$

where $v^{*}=\frac{\phi \mu x_{f}}{K p_{0}} v, x^{*}=\frac{x}{l}$, and $l$ is the length of the mold.

The closed-form solution for the dimensionless mold filling time is given by

$$
t_{\text {fill }}^{*}=\frac{1}{2}
$$

When the mold filling is solved by the CVFEM, assuming the length of the mold is divided into $N$ equal elements, there are $N+1$ control volumes, as shown in Figure 2. The length of each control volume is $1 / N$ for $C_{1}$ through $C V_{N-1}$ and $1 / 2 N$ for $\mathrm{CV}_{0}$ and $\mathrm{CV}_{N}$, respectively.

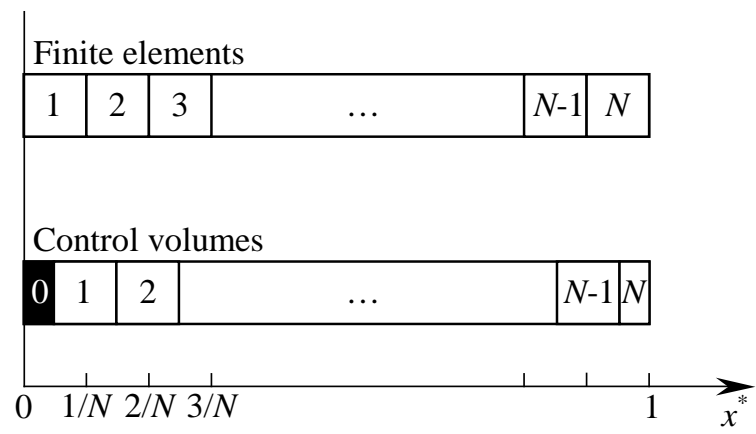

Figure 2: Construction of finite elements and control volumes for channel flow

At the beginning of mold filling, $\mathrm{CV}_{0}$ is filled. The velocities when the flow front is at each control volume from $\mathrm{CV}_{1}$ through $\mathrm{CV}_{N}$ are found using Eqn. 2 as 


$$
\begin{aligned}
& v_{1}^{*}=\frac{\frac{1}{1}}{N}=N \\
& v_{2}^{*}=\frac{1}{\frac{2}{N}}=\frac{N}{2} \\
& \ldots \\
& v_{N-1}^{*}=\frac{1}{\frac{N-1}{N}}=\frac{N}{N-1} \\
& v_{N}^{*}=\frac{1}{\frac{N}{N}}=1
\end{aligned}
$$

The mold filling time in each control volume can be then calculated step-by-step as

$$
\begin{aligned}
& t_{1}^{*}=\frac{\frac{1}{N}}{N}=\frac{1}{N^{2}} \\
& t_{2}^{*}=\frac{\frac{1}{N}}{\frac{N}{2}}=\frac{2}{N^{2}} \\
& \ldots \\
& t_{N-1}^{*}=\frac{\frac{N}{N}}{N-1}=\frac{N-1}{N^{2}} \\
& t_{N}^{*}=\frac{1}{2 N}
\end{aligned}
$$

The total mold filling time is given by

$$
t^{*}=\sum_{i=1}^{N} t_{i}^{*}=\frac{\sum_{i=1}^{N} i}{N^{2}}-\frac{1}{2 N}=\frac{1}{2}
$$

The result from Eqn. 7 is exactly the closed-form solution, which suggests that simulation accuracy for a channel flow is independent of meshing.

\subsection{Radial Flow}

A radial flow is shown in Figure 3. Its closed-form solution is given by (Adams et al. 1988)

$$
t_{\text {fill }}=\frac{\phi \mu}{4 K \Delta p}\left[2 r_{f}^{2} \ln \left(\frac{r_{f}}{r_{0}}\right)-r_{f}^{2}+r_{0}^{2}\right]
$$

where $\Delta p$ is the pressure difference between the injection gate and flow front; $r_{f}$ is the radius of the flow front; and $r_{0}$ is the radius of the injection gate. 


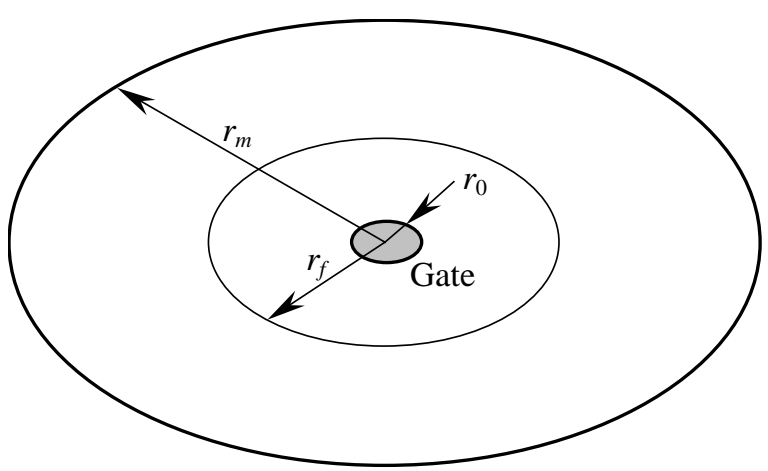

Figure 3: Radial flow

Since $r_{f}>r_{0}, r_{f}$ can be written as $r_{f}=(k+1) r_{0}, k>0$. Based on the boundary conditions of mold filling simulation, the pressure at the gate is $p_{0}$ and at the flow front is zero.

For convenience, the pressure and radius are non-dimensionalized as $r^{*}=\frac{r}{r_{0}}$ and $p^{*}=\frac{p}{p_{0}}$. It is obvious that $r_{0}^{*}=1, r_{f}^{*}=k+1$, and $p_{0}^{*}=1$. Thus, the closedform solution of $p^{*}$ along the $r^{*}$ direction is given as

$$
p^{*}=1-\frac{\ln r^{*}}{\ln r_{f}^{*}}
$$

In CVFEM simulation, if the region from $r_{0}$ to $r_{f}$ is divided into $N$ elements in the $r$ direction, the element size is

$$
h=\frac{r_{f}-r_{0}}{N}=\frac{k r_{0}}{N}
$$

and the dimensionless element size is

$$
h^{*}=\frac{k}{N}
$$

The pressure distribution when $k=80$ and relative errors when $k=20,40$ and 80 using three mesh densities are shown in Figure 4. It is seen that the simulation result approaches the closed-form solution when $h^{*}$ decreases. This is usually called convergence test.
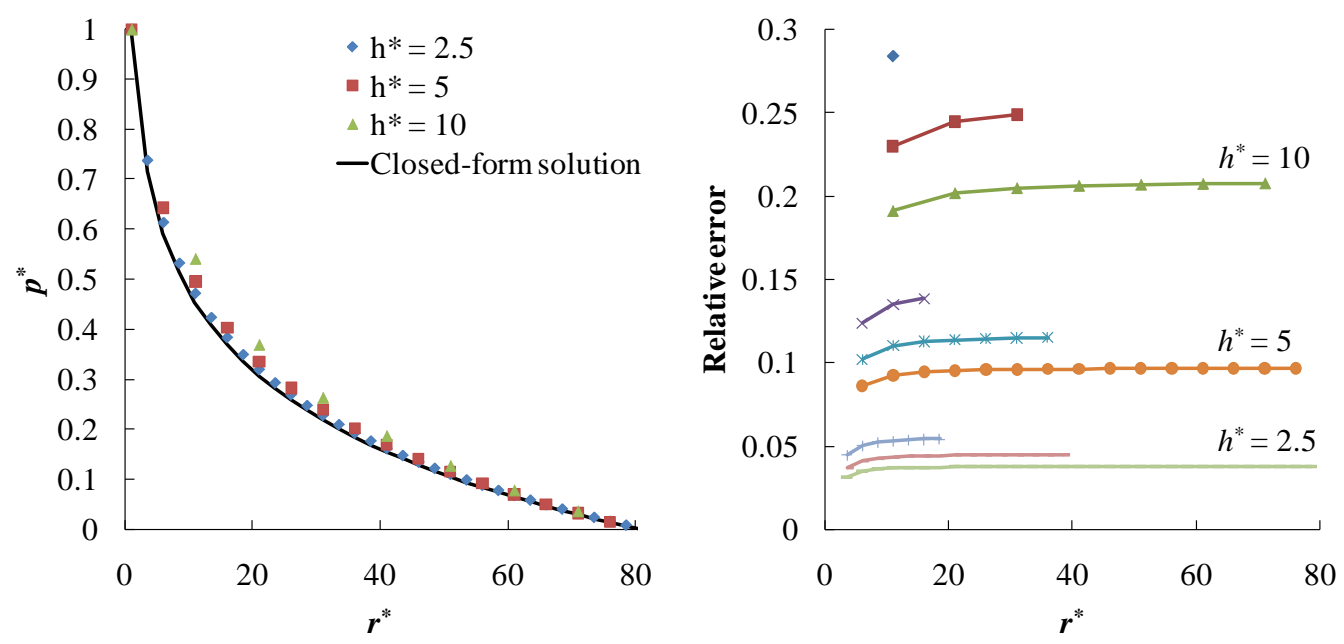
Figure 4: Pressure distribution from simulation when $k=\mathbf{8 0}$ and relative errors when $k=20$, 40 and 80

Besides the validity of simulation, the efficiency is another important issue to be considered. The convergence test shows that the simulation is more accurate when a finer mesh is used. However, as the mesh density increases, the computation time increases tremendously. For example, the computation time vs. number of elements for a typical simulation is as shown in Figure 5. It is seen that when the element size decreases from $10 \mathrm{~mm}$ to $0.625 \mathrm{~mm}$, the computation time increases over four orders of magnitudes. Thus, in order to achieve effective and efficient simulation, the mesh density needs to be properly selected.

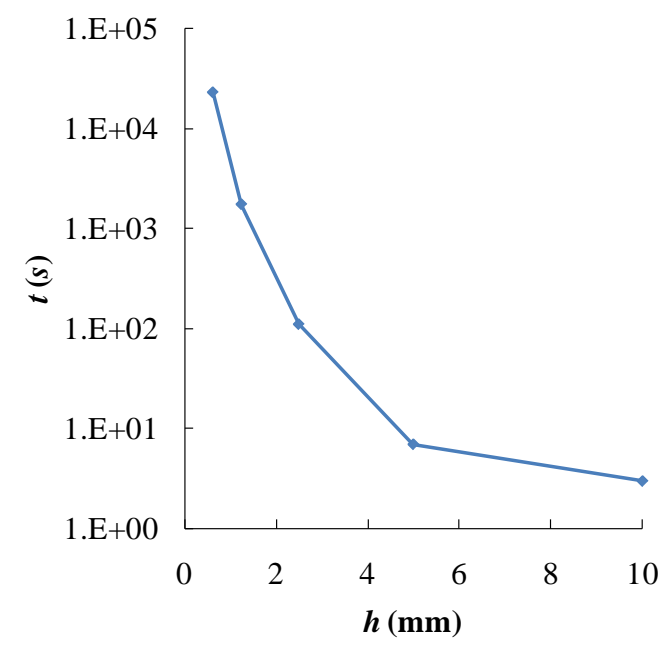

Figure 5: Computation time vs. element size

\section{Non-Uniform Mesh}

Two critical issues in CVFEM are simulation accuracy and computational efficiency. As seen in the previous section, computation time is dependent on the number of elements. One possible way to maintaining good computational efficiency is limiting the number of elements but using non-uniform mesh. When non-uniform meshes are used, the concept of space ratio, $s$ is introduced, which is defined as the ratio of the last and the first elements, i.e. $s=l_{n} / l_{1}$.

For any two consecutive elements $i$ and $i+1(i=1 \ldots n-1)$, the ratio is given by

$$
\frac{l_{i+1}}{l_{i}}=s^{\frac{1}{n-1}}
$$

and $r_{f}$ is given by

$$
r_{f}-1=h_{1} \frac{1-s^{\frac{n}{n-1}}}{1-s^{\frac{1}{n-1}}}
$$

Thus, 


$$
\begin{aligned}
& h_{1}=\left(r_{f}-1\right) \frac{1-s^{\frac{1}{n-1}}}{1-s^{\frac{n}{n-1}}} \\
& h_{2}=\left(r_{f}-1\right) \frac{s^{\frac{1}{n-1}}-s^{\frac{2}{n-1}}}{1-s^{\frac{n}{n-1}}} \\
& \ldots \\
& h_{n}=\left(r_{f}-1\right) \frac{s-s^{\frac{n}{n-1}}}{1-s^{\frac{n}{n-1}}}
\end{aligned}
$$

The pressure at each node can be obtained by solving the following equations.

$$
\frac{1-p_{1}}{h_{1}}\left(1+\frac{h_{1}}{2}\right)=\frac{p_{1}-p_{2}}{h_{2}}\left(1+h_{1}+\frac{h_{2}}{2}\right)=\cdots=\frac{p_{n-1}-p_{n}}{h_{n}}\left(1+h_{1}+h_{2}+\cdots+h_{n-1}+\frac{h_{n}}{2}\right)
$$

i.e.

$$
\begin{aligned}
& \left(1-p_{1}\right)\left(\frac{1}{r_{f}-1} \cdot \frac{1-s^{\frac{n}{n-1}}}{1-s^{\frac{1}{n-1}}}+\frac{1}{2}\right) \\
& =\left(p_{1}-p_{2}\right)\left(\frac{1}{r_{f}-1} \cdot \frac{1-s^{\frac{n}{n-1}}}{s^{\frac{1}{n-1}}-s^{\frac{2}{n-1}}}+\frac{1}{s^{\frac{1}{n-1}}}+\frac{1}{2}\right) \\
& =\cdots \\
& =\left(p_{n-1}-p_{n}\right)\left(\frac{1}{r_{f}-1} \cdot \frac{1-s^{\frac{n}{n-1}}}{s-s^{\frac{n}{n-1}}}+\frac{1}{s}+\frac{1}{s^{\frac{n-2}{n-1}}}+\cdots \frac{1}{s^{\frac{1}{n-1}}}+\frac{1}{2}\right)
\end{aligned}
$$

In this study, a commercial software package ANSYS was used to solve for the pressure distributions. When $k=80$ and $N=8$, the pressure distributions and relative errors from mold filling simulation using different space ratios are shown in Figure 6.

It is seen that when the space ratio is 8 , i.e. $l_{8}=8 l_{1}$, the relative error is $4.27 \%$. If the element spacings are reversed, i.e. $l_{1}=8 l_{8}$, the relative error is $43.56 \%$. The difference is 10 times.

The results show that the region near the injection gate is sensitive to meshing. In order to obtain satisfactory simulation accuracy, fine meshing should be used near the injection gate. 

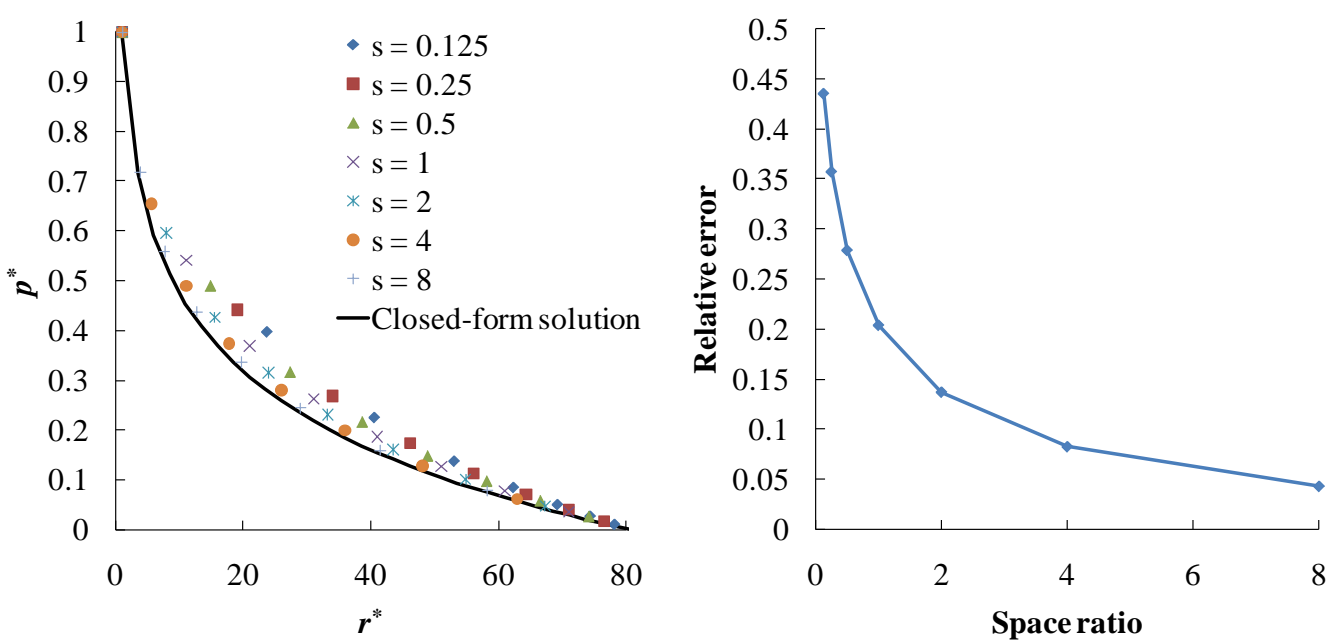

Figure 6: Pressure distributions from simulation using different space ratios

\section{Case Study}

In order to validate this approach, the mold filling of a part as shown in Figure 7 was simulated. Three different meshes: coarse mesh, fine mesh, and smart mesh were used, as shown in Figure 8. The corresponding mold filling processes simulated using these three different meshes is shown in Figure 9 and the results are summarized in Table 1. It is seen that when a mixed mesh which is finer near the gate is used, the relative difference is $0.25 \%$ while a time saving of over $99 \%$ is achieved.

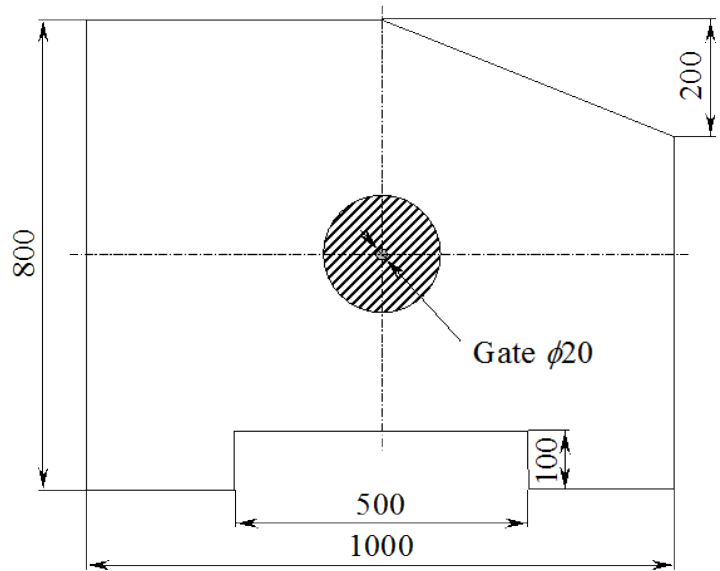

Figure 7: Case study 


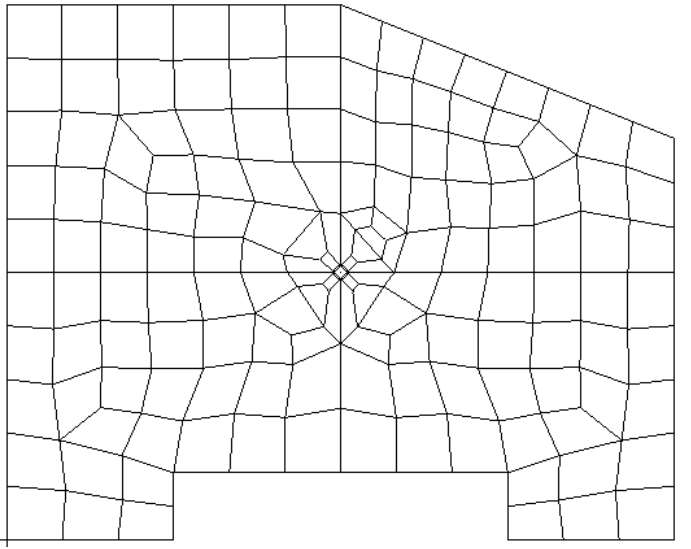

Coarse mesh

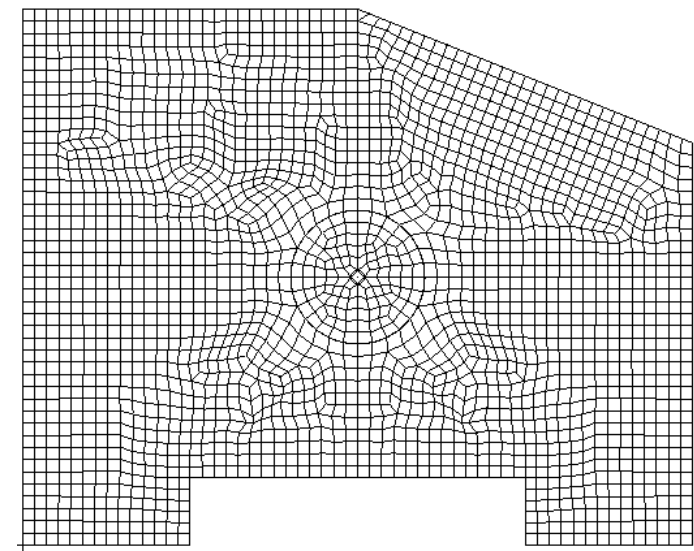

Fine mesh

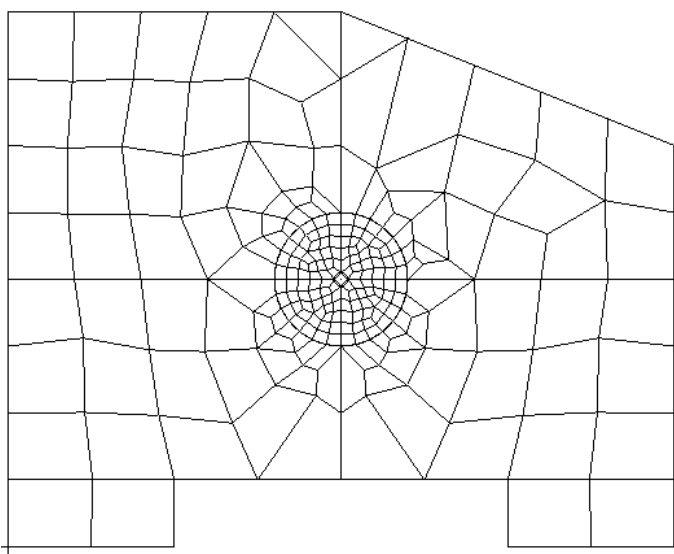

Mixed mesh

Figure 8: Meshes used for mold filling simulation

Table 1: Mold filling simulation using different meshes

\begin{tabular}{lllll}
\hline & $\begin{array}{l}\text { Number of } \\
\text { nodes }\end{array}$ & $\begin{array}{l}\text { Number of } \\
\text { elements }\end{array}$ & $\begin{array}{l}\text { Mold filling time } \\
(\mathrm{s})\end{array}$ & Computation time \\
\hline Coarse mesh & 178 & 152 & 633 & $1 \mathrm{~s}$ \\
Fine mesh & 2032 & 2199 & 786 & $10 \mathrm{~min}$ \\
Smart mesh & 250 & 232 & 788 & $1 \mathrm{~s}$ \\
\hline
\end{tabular}

\section{Conclusions}

Two critical issues of CVFEM are simulation accuracy and computational efficiency, and they are strongly dependent on meshing. In this paper, the influence of meshing on the simulation accuracy is investigated. Both uniform and non-uniform meshes are studied. The results show that for a radial flow, simulation accuracy can be significantly improved by using non-uniform meshes. The region near the injection gate is sensitive to meshing. In order to obtain satisfactory simulation accuracy, fine meshing should be used near the injection gate. The mold filling of a point injection part was simulated using three different meshes. It is shown that using a mixed mesh which is finer near the gate can reduce the computation time by more than $99 \%$ while maintaining the same simulation accuracy. 

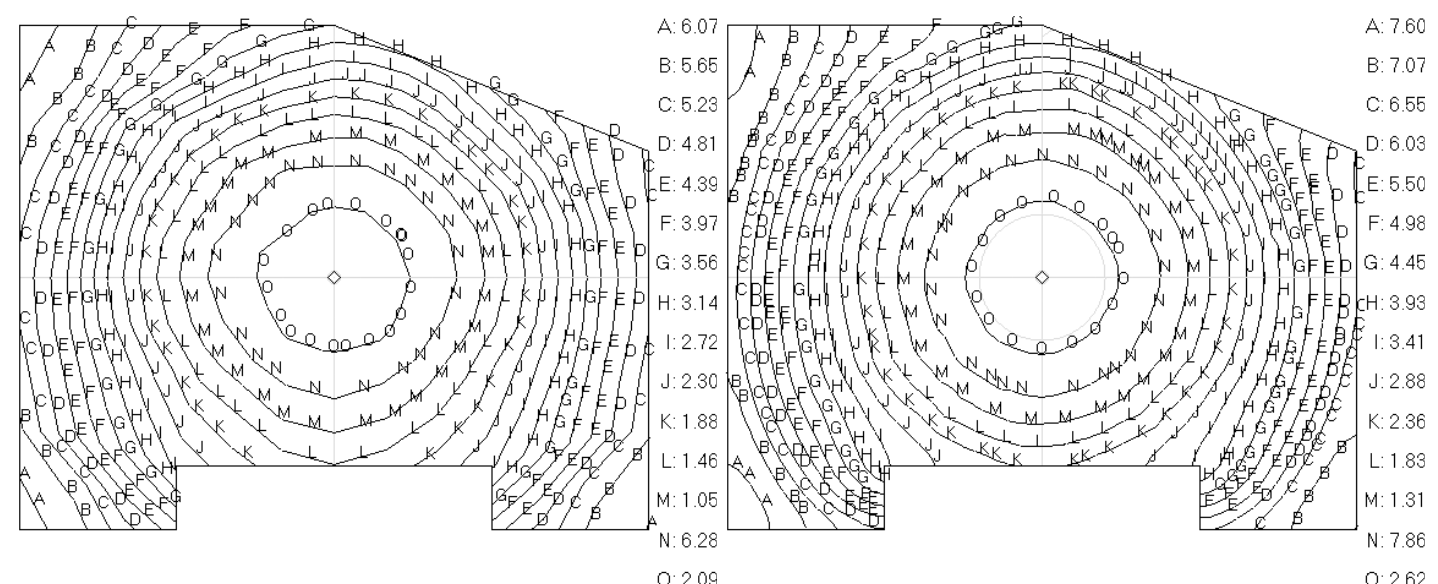

Coarse mesh

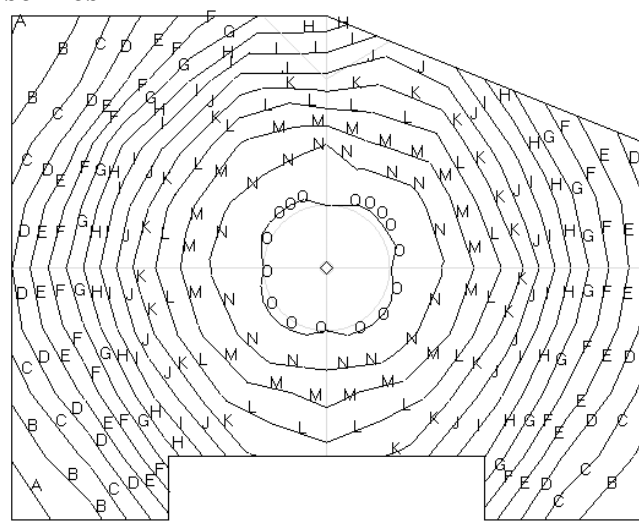

Fine mesh

Mixed mesh

B: $7.01+002$

C. $6.49+002$

D: $5.97+002$

E: $5.45+002$

F: $4.93+002$

G: $4.41+002$

H: $3.89+002$

I: $3.37+002$

J: $2.86+002$

: $2.34+002$

M: $1.30+002$

$\mathrm{N}: 7.79+001$

0: $2.60+001$

Figure 9: Mold filling simulation results using different meshes

\section{References}

Adams, K.L., Russel, W.B., Rebenfeld, L.: Radial penetration of a viscous liquid into a planar anisotropic porous medium. International Journal of Multiphase Flow 14(2), 203-215 (1988)

Béchet, E., Ruiz, E., Trochu, R., Cuilliere, J.-C.: Re-meshing algorithms applied to mould filling simulations in resin transfer moulding. Journal of Reinforced Plastics and Composites 23(1), 17-36 (2004)

Bruschke, M.V., Advani, S.G.: A finite element/control volume approach to mold filling in anisotropic porous media. Polymer Composites 11(6), 398-405 (1990)

Chang, W., Kikuchi, N.: An adaptive remeshing method in simulation of resin transfer molding (RTM) process. Computer Methods in Applied Mechanics and Engineering 112, 41-68 (1994)

Coulter, J.P., Guceri, S.I.: Resin transfer molding: process review, modeling and research opportunities, . In: Proceedings of Manufacturing International '88, Atlanta, GA, USA. 1988

Dong, C.: Injection gate definition for improving the accuracy of liquid composite molding process simulation. Journal of Composite Materials 41(15), 1851-1870 (2007)

Joshi, S.C., Lam, Y.C., and Liu, X.L.: Mass conservation in numerical simulation of resin flow. Composites Part A 31(10), 1061-1068 (2000)

Liu, X.L.: Isothermal flow simulation of liquid composite molding. Composites Part A 31(12), 1295-1302 (2000) 
Modi, D., Šimáček, P., Advani, S.: Influence of injection gate definition on the flow-front approximation in numerical simulations of mold-filling processes. International Journal for Numerical Methods in Fluids 42(11), 1237-1248 (2003)

Osswald, T., Tucker, C.: A boundary element simulation of compression mold filling. Polymer Engineering and Science 28(7), 413-420 (1987)

Šimáček, P., Advani, S.: Gate elements at injection locations in numerical simulations of flow through porous media: applications to mold filling. International Journal for Numerical Methods in Engineering 61(9), 1501-1519 (2004)

Šimáček, P., and Advani, S.G.: Desirable features in mold filling simulations for liquid molding processes. Polymer Composites 25(4), 355-367 (2004)

Um, M.K., Lee, W.I.: A study on mold filling process in resin transfer molding. Polymer Engineering and Science 31(11), 765-771 (1991)

Yoo, Y.E., Lee, W.I.: Numerical simulation of the resin transfer mold filling process using boundary element method. Polymer Composites 17(3), 368-374 (1996)

Young, W.B., Han, K., Fong, L.H., Lee, L.J.: Flow simulation in molds with preplaced fiber mats. Polymer Composites 12(6), 391-404 (1991) 